Artículo científico

Volumen 32(1):178-195. Enero-abril, 2021

e-ISSN 2215-3608, doi:10.15517/am.v32i1.40845

https://www.revistas.ucr.ac.cr/index.php/agromeso

\title{
Raoultella terrigena y Pectobacterium carotovorum en hortalizas en dos provincias de Costa Rica ${ }^{1}$
}

\section{Raoultella terrigena and Pectobacterium carotovorum in vegetables in two provinces of Costa Rica}

\author{
Diego Cubero-Agüero², Laura Brenes-Guillén ${ }^{2}$, Daniela Vidaurre-Barahona², Lorena Uribe-Lorío ${ }^{2,3}$
}

1 Recepción: 27 de febrero, 2020. Aceptación: 2 de julio, 2020. Este trabajo formó parte de la tesis de Licenciatura realizada por el primer autor, Universidad de Costa Rica, Costa Rica.

2 Universidad de Costa Rica, Centro de Investigación en Biología Celular y Molecular, 11501-2060, San Pedro de Montes de Oca, San José, Costa Rica.diego.cuberoaguero@ucr.ac.cr (autor para la correspondencia, https://orcid.org/0000-0001-9205-5647), laura.brenesguillen@ucr. ac.cr (https://orcid.org/0000-0002-7185-4084), daniela.vidaurre@ucr.ac.cr (https://orcid.org/0000-0002-9221-0560), lorena.uribe@ucr.ac.cr (https://orcid.org/0000-0002-6023-0997).

3 Universidad de Costa Rica, Escuela de Agronomía, 1501-2060, San Pedro de Montes de Oca, San José, Costa Rica.

\section{Resumen}

Introducción. La pudrición blanda en los cultivos es causada por un grupo de bacterias capaces de secretar enzimas que degradan la pectina de la pared celular de las plantas y ocasionan pérdidas económicas importantes en la agricultura a nivel mundial. En Costa Rica existe poca información acerca de la distribución, hospederos y diversidad genética de los agentes causales de esta enfermedad. Objetivo. Identificar la presencia de bacterias causantes de pudrición blanda en hortalizas de la zona de Cartago y Alajuela con análisis moleculares, enzimáticos y técnicas de patogenicidad. Materiales y métodos. El estudio se llevó a cabo entre julio y octubre de 2017 en Cartago y Alajuela, Costa Rica. Se recolectaron plantas con síntomas de pudrición blanda en: chile dulce (Capcicum annum), hojas y bulbos de cebolla (Allium cepa), plantas de zucchini (Cucurbita pepo), frutos de tomate (Solanum lycopersicum) y plantas de papa (Solanum tuberosum). Se realizaron aislamientos bacterianos en un medio selectivo de cristal violeta y pectato (CVP). Se efectuaron ensayos de reacción hipersensible (RH), los aislamientos se inocularon en hojas de chile dulce y se llevaron a cabo pruebas de patogenicidad (postulados de Koch) para las bacterias positivas. Los aislamientos se identificaron molecularmente mediante el gen ARN-Ribosomal 16S. Resultados. Se aislaron cinco bacterias con actividad pectinolítica: Pt1-A, 6-M2, Ech2A, CfspA y Cfsab. Según el análisis de patogenicidad, las cepas CfspA, Cfsab y Ech2A fueron causantes de pudrición blanda en chile. No se logró reproducir los síntomas en cebolla y papa. De acuerdo con la identificación molecular, las cepas CfspA y Cfsab se clasificaron dentro del clado de Pectobacterium carotovorum, mientras que Ech2A se clasificó como Raoultella terrigena. Conclusión. Las bacterias Raoultella terrigena y Pectobacterium carotovorum se consideraron los agentes causales de pudrición blanda del chile en las zonas de Cartago y Alajuela, respectivamente.

Palabras clave: pudrición blanda, fitopatología, análisis molecular. 


\begin{abstract}
Introduction. Soft rot in crops is caused by a group of bacteria capable of secreting enzymes that degrade pectin in the cell wall of the plants and causes significant economic losses in agriculture worldwide. In Costa Rica, there is few data available about the distribution, hosts, and genetic diversity of the causal agents of disease. Objective. To identify the presence of bacteria causing soft rot in vegetables located in Cartago and Alajuela using molecular and enzymatic analysis, and pathogenicity techniques. Materials and methods. The study was carried out between July and October 2017 in Cartago and Alajuela, Costa Rica. Plants with soft roth symptoms were collected: sweet chili (Capcicum annum), onion leaves and bulbs (Allium cepa), zucchini plants (Cucurbita pepo), tomato fruits (Solanum lycopersicum), and potato plants (Solanum tuberosum). Bacterial isolation was performed on a selective violet crystal and pectate (CVP) medium. Hypersensitive reaction (RH) tests were performed, the isolates were inoculated into sweet chili leaves and pathogenicity tests (Koch's postulates) were carried out for the positive bacteria. The isolates were identified by the 16S RNA-Ribosomal gene. Results. Five bacteria with pectinolytic activity were isolated: Pt1-A, 6-M2, Ech2A, CfspA, and Cfsab. According to the pathogenicity analysis, CfspA, Cfsab, and Ech2A strains were causal agents of soft rot in chile. It was not possible to reproduce the symptoms in onion and potato. According to the molecular identification, CfspA and Cfsab strains were classified within the Pectobacterium carotovorum clade, while Ech2A was classified as Raoultella terrigena. Conclusion. Raoultella and Pectobacterium carotovorum were considered the causal agents of soft rotting of chili in the areas of Cartago and Alajuela respectively.
\end{abstract}

Keywords: soft rot, phytopathology, molecular analysis.

\title{
Introducción
}

En Costa Rica, la horticultura es una de las actividades agrícolas más importantes y la mayor parte de la producción se localiza en la región central del país, principalmente en las faldas de los volcanes Irazú, Turrialba y Poás, pertenecientes a las provincias de Cartago y Alajuela (Wei-Salas \& Durán-Quirós, 2015). Se lleva a cabo en campo abierto, bajo condiciones ambientales de alta humedad relativa, precipitaciones entre los 0 y $100 \mathrm{~mm}$ en meses secos y entre los 200 a $500 \mathrm{~mm}$ en meses lluviosos y con temperaturas promedio entre los 16 y $20^{\circ} \mathrm{C}$, lo cual podría resultar adverso para los cultivos y favorable para organismos fitopatógenos como bacterias, hongos, virus y nemátodos (Agrios, 2005), ya que estos interactúan con las plantas y pueden causar enfermedades económicamente importantes (Arauz, 2011).

Una de las principales enfermedades en la producción hortícola es la podredumbre blanda. Los principales agentes causales de esta enfermedad corresponden a bacterias pectinolíticas (Czajkowski et al., 2015) pertenecientes a los géneros Pseudomonas, Burkholderia, Pantoea, Enterobacter, Klebsiella, Leuconostoc, Bacillus, Xanthomonas, Clostridium, Erwinia, Pectobacterium y Dickeya (Nguyen-the y Carlin, 1994; Charkowski et al., 2014), estos dos últimos son los géneros que causan los síntomas mayores asociados a la sintomatología (Charkowski, 2018).

Pectobacterium y Dickeya son conocidos en la actualidad como enterobacterias de la podredumbre blanda (Soft Rot Enterobacteriacea, SRE). A diferencia de otros géneros mencionados anteriormente, se considera que estos poseen mayor agresividad y efectividad para causar la enfermedad, debido a que son capaces de sintetizar un complejo enzimático más amplio. Estas características les permite degradar la pectina presente en los tejidos vegetales y afectar directamente la firmeza tisular de las plantas (Hugouvieux-Cotte-Pattat et al., 2014).

La podredumbre blanda bacteriana (BSR por sus siglas en inglés, Bacterial soft rot) provocada por las SRE, es considerada una de las causas de mayores pérdidas económicas en la agricultura alrededor del mundo (Charkowski 
et al., 2014). Se ha diagnosticado en muchas regiones agrícolas y afecta frutales, cereales, vegetales y ornamentales (Wright et al., 2005; Kim et al., 2007; Ma et al., 2007; Bhat et al., 2010; Pu et al., 2012; Zhang et al., 2014). La sintomatología se puede observar desde la emergencia de los cultivos hasta la etapa de almacenamiento, en donde se registra la mayor pérdida, debido a que la descomposición de algunos vegetales puede propagar las bacterias rápidamente (Bhat et al., 2010).

La mayoría de investigaciones sobre la BSR se han realizado en papa (Solanum tuberosum) (Charkowski et al., 2014), por ser uno de los cultivos más importantes a nivel mundial (Singh \& Kaur, 2016). Diferentes especies de Pectobacterium y Dickeya se han mencionado como causales de la pudrición blanda o pie negro como es llamada popularmente (Ngadze et al., 2010; Pitman et al., 2008; van-der-Merwe et al., 2010; Waleron et al., 2015). La sintomatología se ha reportado también en diversas familias como Solanaceae, Cucurbitaceae, Brassicaceae, Alliaceae y Araceae (Ma et al., 2007). En Costa Rica, se ha analizado el desarrollo y combate de E. carotovorum subsp. atroseptica (hoy Pectobaterium atrosepticum, Gardan et al., 2003) en tubérculos de papa (Herrera \& González, 1977) mientras que, en el año 1979, se identificó E. chrysanthemi en el cultivo de piña como causante de BSR (Chinchilla et al., 1979). Recientemente, se identificó la bacteria Enterobacter hormaechei como posible agente causal de la pudrición blanda en los tallos de pitahaya (Hylocereus costaricensis) (Sánchez et al., 2019). No obstante, en ninguno de estos estudios se han realizado análisis moleculares detallados de los agentes causales con el fin de confirmar su identificación. Existe muy poca o nula información actualizada en cuanto a la distribución, hospederos y diversidad genética de las bacterias que causan la podredumbre blanda en nuestro país.

El objetivo de este estudio fue identificar con análisis moleculares, enzimáticos y técnicas de patogenicidad, la presencia de bacterias causantes de pudrición blanda en hortalizas de la zona de Cartago y Alajuela.

\section{Materiales y métodos}

Las diferentes pruebas y actividades de laboratorio se llevaron a cabo en el laboratorio de Microbiología Ambiental del Centro de Investigación en Biología Celular y Molecular (CIBCM), de la Universidad de Costa Rica. Por otro lado, para los ensayos llevados a cabo en invernadero, se utilizó el invernadero del área de Microbiología de Suelos del Centro de Investigaciones Agronómicas de la Universidad de Costa Rica.
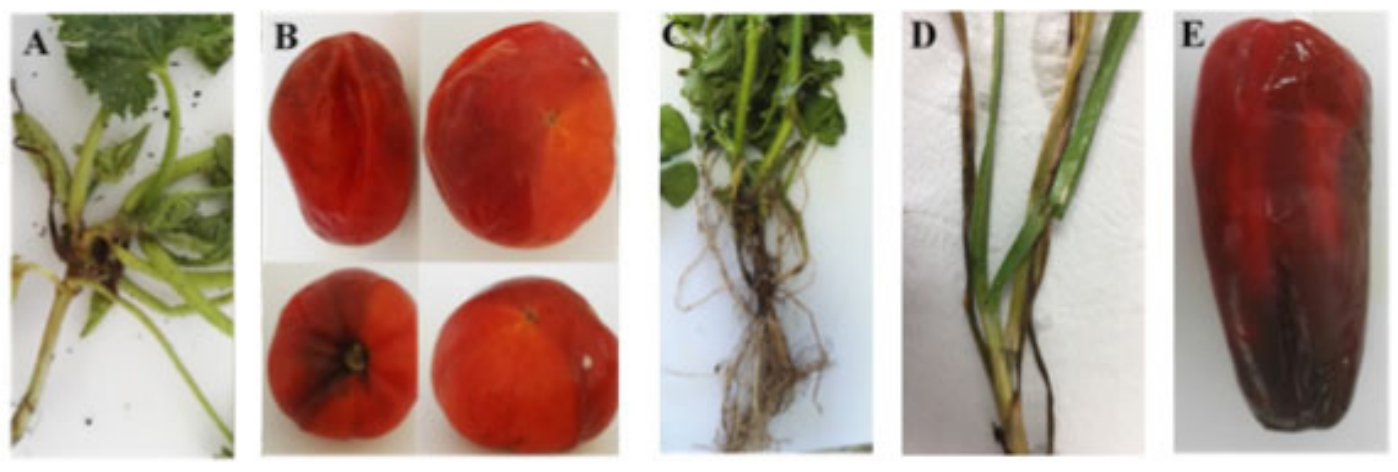

Figura 1. Sintomatología de plantas y frutos hospederos de los aislamientos bacterianos. Fotografías tomadas en el laboratorio de Microbiología Ambiental del Centro de Investigación en Biología Celular y Molecular (CIBCM), de la Universidad de Costa Rica. A. Tallo de zucchini (C. pepo) Cartago, B. Frutos de tomate (S. lycopersicum) Cartago, C. Tallo de papa (S. tuberosum), Cartago D. Hojas de cebolla (A. серa), Cartago, E. Frutos de chile dulce (C. annuиm), Cartago y Alajuela, Costa Rica. 2017

Figure 1. Symptomatology of the host plants of bacterial isolates. The photographs were taken in the Environmental Microbiology Laboratory (CIBCM), University of Costa Rica. A. Zucchini stem (C. pepo), Cartago, B. Tomato fruits (S. lycopersicum), Cartago, C. Potato stalk (S.tuberosum), Cartago, D. Onion leaves (A. сера), Cartago, E. Sweet chili fruits (C. annиum), Cartago and Alajuela, Costa Rica. 2017. 


\section{Recolección de las muestras}

Durante los meses de julio y octubre del año 2017, se colectaron plantas con síntomas de pudrición blanda en fincas hortícolas de las provincias de Cartago y Alajuela, Costa Rica. En la zona de Oreamuno (Cartago), se tomaron muestras de frutos de chile dulce (Capcicum annum), hojas y bulbos de cebolla (Allium cepa), plantas de zucchini (Cucurbita pepo) y frutos de tomate (Solanum lycopersicum), en Juan Viñas (Cartago) plantas de tomate y en Tierra Blanca (Cartago) de papa (Solanum tuberosum) respectivamente. En la Garita de Alajuela, se colectaron frutos de chile dulce (Figura 1).

\section{Aislamiento bacteriano}

Se realizó un lavado superficial de las muestras con agua estéril y se extrajeron tres secciones de tejido de la zona de avance de las lesiones, las cuales se desinfectaron mediante inmersión en hipoclorito de sodio al $1 \%$ durante dos minutos, seguido de tres lavados con agua destilada estéril.

Cada muestra se inoculó en tres tubos de ensayo que contenían agua estéril, caldo nutritivo y medio líquido de pectina (polipectato de sodio al $75 \%$, de $\left(\mathrm{NH}_{4}\right)_{2} \mathrm{SO}_{4}$ al $10 \%$, de $\mathrm{Mg}_{2} \mathrm{SO}_{4} 7 \mathrm{H}_{2} \mathrm{O}$ al $5 \%$, y de $\mathrm{K}_{2} \mathrm{HPO}_{4}$ al $10 \%$ ) (Bdliya \& Langerfeld, 2005). Los tubos con agua se agitaron durante cinco minutos a 50 r.p.m. para desprender las células bacterianas del tejido y, posteriormente, se cultivó la suspensión obtenida en placas con agar MacConkey (OXOID), las cuales se incubaron por $24 \mathrm{~h} \mathrm{a} 28{ }^{\circ} \mathrm{C}$. El tejido inoculado en caldo nutritivo y medio líquido de pectina se mantuvo en condiciones de anaerobiosis con aceite mineral y se incubó durante $24 \mathrm{~h}$ a $28^{\circ} \mathrm{C}$, posterior a este periodo, se cultivaron en placas con agar MacConkey.

Después del período de incubación, las colonias observadas en cada placa se clasificaron según morfología y pruebas como tinción de Gram, y se replicaron hasta obtener un cultivo puro. Posteriormente, se realizaron análisis de oxidasa, coloración en MacConkey, crecimiento anaeróbico y pectinólisis en papa para seleccionar los aislamientos Gram negativos entéricos con el propósito de proseguir con los análisis de reacción de hipersensibilidad y patogenicidad.

\section{Prueba de reacción de hipersensibilidad $(\mathrm{RH})$}

Para la prueba de reacción de hipersensibilidad $(\mathrm{RH})$ la metodología empleada se basó en los protocolos utilizados por Bonas et al. (1991), Klement (1963) y Umesha et al. (2008). En el laboratorio, las cepas se cultivaron en medio de agar nutritivo (OXOID) y se incubaron a $30^{\circ} \mathrm{C}$ por $24 \mathrm{~h}$. Una vez transcurrido el tiempo, se prepararon suspensiones bacterianas en agua estéril a una concentración de $9 \times 10^{8} \mathrm{UFC} \mathrm{ml}^{-1}$ (unidades formadoras de colonias), comparada con un patrón de turbidez McFarland número tres (McFarland, 1907). La planta indicadora utilizada para realizar esta prueba fue chile dulce (Capsicum annuum) (Ritchie, 2000). Cada cepa se inoculó en cinco hojas, donde se realizaron cuatro puntos de inoculación en cada una, tres de las cepas bacterianas evaluadas y una de agua estéril como control negativo. Se aplicó aproximadamente $50 \mu l$ por punto por medio de presión en el envés de las hojas, con una jeringa sin aguja, el proceso de inoculación se llevó a cabo en el invernadero donde se encontraban las plantas.

Las plantas inoculadas se mantuvieron en el invernadero donde la luz y temperatura eran variables de acuerdo a las condiciones ambientales. Se realizaron observaciones durante siete días consecutivos, se inició a las $24 \mathrm{~h}$ después de la inoculación y se evaluó el posible desarrollo de clorosis o necrosis alrededor del punto inoculado, lo cual es un indicativo de un resultado positivo para la RH. En el caso de presentarse la RH en la hoja después de la inoculación, la bacteria causante de la reacción se consideró como fitopatógena, de lo contrario se descartó para las pruebas posteriores de actividad pectinolítica. 


\section{Actividad pectinolítica en medio cristal violeta y pectato (CVP)}

Se analizó la actividad pectinolítica de los aislamientos bacterianos que causaron RH bajo condiciones de laboratorio. Las bacterias seleccionadas se cultivaron en agar nutritivo (OXOID) y se incubaron a $30{ }^{\circ} \mathrm{C}$ durante 24

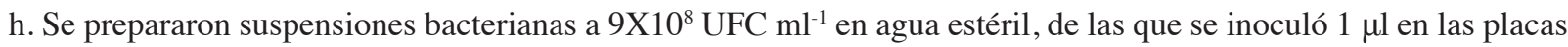
con CVP y se incubaron a $30{ }^{\circ} \mathrm{C}$ durante cuatro días. El medio de CVP se preparó según lo descrito por Schaad et al. (2001), donde se utilizó como fuente de pectina el ácido polianhidrogalacturónico (Sigma-Aldrich Ref. P3850). Se evaluó la presencia o ausencia de cavidades en los puntos donde fueron inoculados los aislamientos, lo cual se determinó como la capacidad de las bacterias de metabolizar la pectina presente en el medio.

\section{Postulados de Koch para aislamientos seleccionados}

La verificación de los postulados de Koch se realizó para aquellos aislamientos que causaron la RH en las plantas de chile dulce y que provocaron cavidad en el medio de CVP. Este análisis consistió en la inoculación de las bacterias en hospederos sanos, con el objetivo de verificar si existía una relación entre los diferentes aislamientos con la pudrición blanda en las plantas (Byrd \& Segre, 2016; Volcy, 2008). La metodología utilizada para cada uno se detalla a continuación:

\section{Frutos de chile dulce}

Para la inoculación, las cepas se cultivaron en medio de agar nutritivo (OXOID) y se incubaron a $30{ }^{\circ} \mathrm{C}$ por $24 \mathrm{~h}$. Una vez transcurrido el tiempo de incubación, se tomaron colonias aisladas para preparar suspensiones bacterianas a una concentración de $9 \times 10^{8} \mathrm{UFC} \mathrm{ml}^{-1}$, comparada con el patrón de turbidez McFarland número tres. Se utilizaron frutos sanos, los cuales se desinfectaron con una solución de hipoclorito de sodio al $2 \%$ durante tres minutos dentro de la cámara de flujo laminar, posteriormente, se removió el exceso con tres lavados con agua destilada estéril y se dejaron secar.

Se realizaron cuatro hoyos en el pericarpio del fruto a una profundidad aproximada de $5 \mathrm{~mm}$ y se inocularon 30 $\mu \mathrm{l}$ de suspensión bacteriana en dos o tres y el mismo volumen de agua estéril en los restantes. Los frutos inoculados se colocaron en cámaras de alta humedad (cajas plásticas con toallas humedecidas con agua estéril) dentro de incubadoras en el laboratorio, se incubaron a $30{ }^{\circ} \mathrm{C}$ y se realizó evaluaciones diarias.

\section{Plantas de papa}

Se sembró semilla de papa (tubérculos con brotación) en bandejas de 72 celdas con suelo estéril. A los 15 días de emergidas, las plantas (brotes con $10 \mathrm{~cm}$ de altura aproximadamente) se trasladaron al invernadero del Centro de Investigaciones Agronómicas (CIA) de la Universidad de Costa Rica. Se trasplantaron a potes de 21 a media capacidad de suelo y se realizó una inoculación de $5 \mathrm{ml}$ de suspensión bacteriana a una concentración de 9X108 UFC, comparada con un patrón de turbidez McFarland número tres.

La inoculación se realizó en el adobe formado por la celda de la bandeja. Posteriormente, los potes se rellenaron con suelo hasta máxima capacidad y se colocaron dentro de una caja plástica con el propósito de aumentar la humedad relativa. Para esta prueba se utilizaron tres que contenían tres plantas cada uno. Dos se inocularon con la bacteria y uno con agua estéril como control negativo.

Después de la inoculación, las plantas se mantuvieron en el invernadero a temperatura no controlada, con temperaturas promedio de $28^{\circ} \mathrm{C}$ y se colocaron dentro de una caja plástica transparente para aumentar la humedad 
relativa, con el objetivo de mejorar las condiciones requeridas para la infección bacteriana. Las evaluaciones se realizaron diariamente durante veintidós días.

\section{Cebolla}

Se utilizaron bulbos maduros de cebolla, los cuales se indujeron bajo condiciones de alta humedad y temperatura para el desarrollo de hojas y raíces, posteriormente, se plantaron en potes con $200 \mathrm{ml}$ con suelo. Una vez que las plantas presentaron entre cuatro a cinco hojas, se inocularon con una suspensión bacteriana a una concentración de $10 \mathrm{X}^{8} \mathrm{UFC} \mathrm{ml}^{-1}$, la cual se comparó con el patrón McFarland número tres.

Se utilizaron dos métodos de inoculación, el primero consistió en agregar $5 \mathrm{ml}$ de suspensión bacteriana en el suelo donde fue sembrado el bulbo, y el segundo en inocular el mismo volumen entre la base de las hojas y el bulbo. Después de la inoculación, las plantas se trasladaron al invernadero (condiciones de temperatura no controladas, temperatura promedio $28^{\circ} \mathrm{C}$ ) y se colocaron dentro de bolsas plásticas transparentes para aumentar la humedad relativa. Las evaluaciones se realizaron diariamente durante 15 días.

\section{Extracción de ADN y análisis filogenéticos}

\section{Comparación de cepa inoculada con la reaislada en los postulados de Koch}

Con el propósito de determinar que el organismo inoculado correspondiera con el recuperado en los postulados de Koch, se llevó a cabo la comparación molecular y morfológica de los aislamientos. Se realizó una tinción de Gram y visualización de los cultivos en placas de Petri con agar nutritivo. Además, las cepas inoculadas y las recuperadas se compararon mediante la amplificación de la región intergénica 16S-23S del gen rARN (ITS) con los imprimadores G1 (5'-GAAGTCGTAACAAAGG-3') y L1 (5'-CAAGGCATCCACCGT-3') (Jensen et al., 1993). Con el patrón de bandeo obtenido se realizó un análisis UPGMA (método de agrupamiento de pares no ponderados con media aritmética) para comparar la similitud.

\section{Análisis filogenético del gen $16 S$ ARNr para las cepas causantes de pudrición blanda}

Se realizó la extracción de ADN a partir de una colonia de cada aislamiento causante de la pudrición blanda con el Kit comercial Instagen de Bio-Rad, de acuerdo con las instrucciones del fabricante. Posteriormente, se amplificó el gen 16S ARN ribosomal mediante reacción en cadena de la polimerasa (PCR) utilizando los imprimadores 27F (5'-AGAGTTTGATCCTGGCTCAG-3') y 907R (5'-CCGTCAATTCMTTTRAGTTT-3') (Lane, 1991), 1X de Dream taq buffer con $\mathrm{MgCl}_{2} 25 \mathrm{mM}, 0,2 \mathrm{mM}$ de DNTPs mix (Thermo Fisher Scientific), 1,25 mM de $\mathrm{MgCl}_{2} 25$

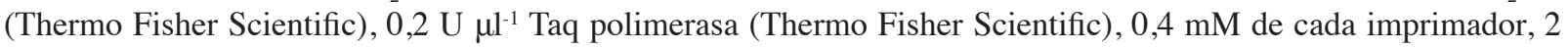
$\mu \mathrm{l}$ de ADN y agua estéril para completar el volumen de reacción de $50 \mu \mathrm{l}$.

Se utilizó un termociclador Veriti 95 (Applied Biosystems) con el siguiente programa: un paso inicial de desnaturalización de 4 min a $94{ }^{\circ} \mathrm{C}$, seguido de 30 ciclos de desnaturalización a $94{ }^{\circ} \mathrm{C}$ por $30 \mathrm{~s}$, amplificación a $55^{\circ} \mathrm{C}$ por $30 \mathrm{~s}$ y elongación a $72{ }^{\circ} \mathrm{C}$ por 1,5 min con un periodo de elongación de 7 min (Fontecha, 2003). Los productos de PCR se analizaron en un gel de agarosa al $1 \%$. Los productos de PCR se enviaron a la compañía Macrogen Inc. (Korea) para su secuenciación.

Las secuencias se editaron con el software Bioedit versión 7.0.5.3 (Hall, 1999). Se utilizó la herramienta BLAST del Centro Nacional de Información en Biotecnología (NCBI) para compararlas con secuencias de referencias disponibles en la base de datos. Seguidamente, se realizó un alineamiento con el programa Mega 7 v7.0.26 (Kumar et al., 2016) y se analizó filogenéticamente según probabilidades Bayesianas con Mr Bayes v3.2.5 
(Ronquist et al., 2012). El árbol fue obtenido por la adición de 1000 réplicas y se visualizó con FigTree v1.4.3. Los intervalos de confianza se calcularon en porcentaje.

\section{Resultados}

En total se obtuvieron 31 aislamientos bacterianos de las diferentes plantas analizadas. De estos aislamientos, $29 \%$ provinieron de frutos de chile dulce, $3 \%$ de cebolla, $19 \%$ de zucchini, $19 \%$ de tomate (frutos y planta) y $29 \%$ de plantas de papa (Cuadro 1).

Cuadro 1. Resultados para las pruebas de reacción hipersensible (RH) realizadas en plantas de chile dulce (C. annuum) en invernadero y cavidad en medio de cristal violeta y pectado (CVP) realizado en laboratorio, realizados con las cepas que fueron aisladas a partir de plantas con síntomas de pudrición blanda. San José, Costa Rica 2019. Invernadero de Microbiología de Suelos (CIA) y Laboratorio de Microbiología Ambiental (CIBCM), Universidad de Costa Rica, San José, Costa Rica 2019.

Table 1. Results for hypersensitive reaction (HR) tests developed in greenhouse plants of sweet pepper (C. annuum) and cavity in half crystal violet pectate (CVP) developed in the laboratory performed with strains that were isolated from plants with symptoms of soft rot. Soil Microbiology Greenhouse (CIA) and Environmental Microbiology Laboratory (CIBCM), University of Costa Rica, San José, Costa Rica 2019.

\begin{tabular}{|c|c|c|c|c|c|}
\hline Cepas & Hospedero & Órgano & Procedencia & RH & CVP \\
\hline 6-M2 & Cebolla & Seudotallo & Cartago & ++ & ++ \\
\hline CF1 & Chile & Fruto & Cartago & + & + \\
\hline $\mathrm{CF} 2$ & Chile & Fruto & Cartago & + & + \\
\hline $\mathrm{CF} 3$ & Chile & Fruto & Cartago & + & + \\
\hline CF4 & Chile & Fruto & Cartago & + & + \\
\hline CF5 & Chile & Fruto & Cartago & + & + \\
\hline Cfsaa & Chile & Fruto & Alajuela & ++ & + \\
\hline CfspA & Chile & Fruto & Alajuela & +++ & +++ \\
\hline Csfab & Chile & Fruto & Alajuela & +++ & +++ \\
\hline $\mathrm{ECH} 2 \mathrm{~A}$ & Chile & Fruto & Cartago & ++ & ++ \\
\hline Pt1-A & Papa & Tallo & Tierra Blanca & ++ & ++ \\
\hline Pt1-B & Papa & Tallo & Tierra Blanca & ++ & + \\
\hline Pt4-1 & Papa & Tallo & Tierra Blanca & + & + \\
\hline Pt4-2 & Papa & Tallo & Tierra Blanca & ++ & + \\
\hline Pt4-3 & Papa & Tallo & Tierra Blanca & - & - \\
\hline Pt4-4 & Papa & Tallo & Tierra Blanca & - & - \\
\hline Pt4-5 & Papa & Tallo & Tierra Blanca & - & - \\
\hline Pt4-6 & Papa & Tallo & Tierra Blanca & ++ & - \\
\hline Pt4-7 & Papa & Tallo & Tierra Blanca & ++ & - \\
\hline Tf_CP_1 & Tomate & Fruto & Cartago & + & - \\
\hline Tf_CP_2 & Tomate & Fruto & Cartago & - & - \\
\hline $\mathrm{t} \_\mathrm{CP}-1$ & Tomate & Tallo & Juan Viñas & - & - \\
\hline Tt_CP-2 & Tomate & Tallo & Juan Viñas & ++ & + \\
\hline Tt_CP-3 & Tomate & Tallo & Juan Viñas & - & - \\
\hline Tt_CP-4 & Tomate & Tallo & Juan Viñas & - & - \\
\hline Zt_CP_1 & Zucchini & Tallo & Cartago & ++ & - \\
\hline Zt_CP_2 & Zucchini & Tallo & Cartago & - & - \\
\hline $\mathrm{Zt1}$ & Zucchini & Tallo & Cartago & + & - \\
\hline $\mathrm{Zt} 2$ & Zucchini & Tallo & Cartago & ++ & - \\
\hline $\mathrm{Zt} 3$ & Zucchini & Tallo & Cartago & - & - \\
\hline $\mathrm{Zt} 4$ & Zucchini & Tallo & Cartago & + & - \\
\hline
\end{tabular}




\section{Prueba de reacción de hipersensibilidad (RH)}

Se utilizó una escala según el grado de lesión que presentó la RH, símbolo - cuando no hubo efecto, + para clorosis baja, ++ para clorosis alta y +++ cuando se presetentó necrosis (Figura 2). De los 31 aislamientos obtenidos, nueve no causaron RH (-), de los cuales, dos provenían de zucchini, cuatro de tomate y tres de papa. En nueve cepas se observó la mínima reacción de RH (+), cinco de estas se obtuvieron a partir de chile. Once cepas presentaron RH grado ++ y solamente dos cepas aisladas a partir de fruto de chile, CfspA y Csfab, provocaron necrosis en el punto de inoculación.
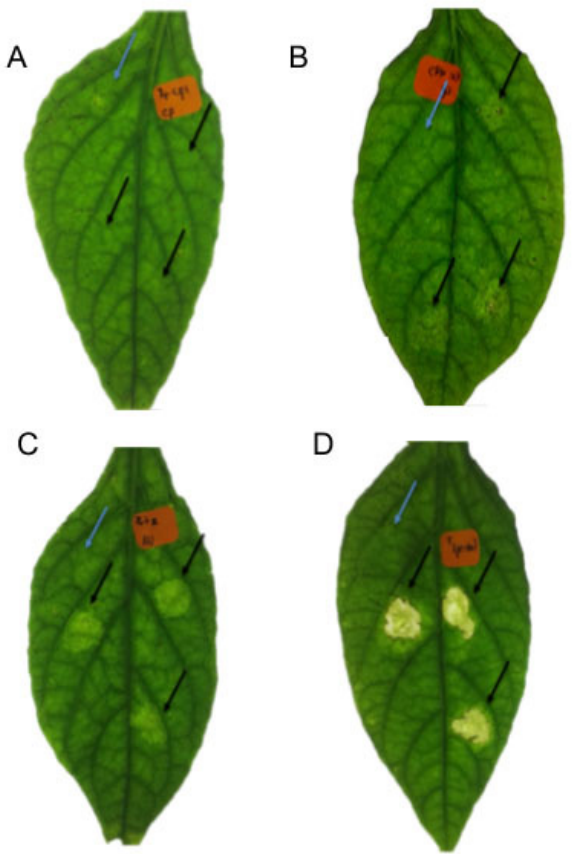

Figura 2. Pruebas de reacción hipersensible $(\mathrm{RH})$ en hojas de chile dulce (Capsicum annuum), provocada por los aislamientos bacterianos inoculados. La imagen muestra los grados en los que se observó la reacción en los puntos de inoculación de las distintas cepas. Las flechas azules corresponden al punto en el cual se inoculó agua estéril mientras que las flechas negras corresponden a los puntos donde fueron inoculadas las cepas bacterianas. A. Grado -, B. Grado + C. Grado ++ y D. Grado +++. Invernadero de Microbiología de Suelos (CIA), Universidad de Costa Rica. San José, Costa Rica, 2019.

Figure 2. RH reactions in sweet pepper leaves (Capsicum апnиит) caused by the bacterial isolates inoculated. The image shows the severity range of the reaction observed at the inoculated points. The blue arrows correspond to the point inoculated with sterile water while the black arrows, with the bacterial strain. A. Grade -, B. +, C. Grade ++ and D. Grade +++. Soil Microbiology Greenhouse (CIA), University of Costa Rica, San Jose, Costa Rica, 2019.

\section{Actividad pectinolítica en medio cristal violeta y pectato (CVP)}

Para la actividad pectinolítica también se asignó una escala, - cuando no hubo crecimiento, + cuando hubo crecimiento sin cavidad, ++ crecimiento con cavidad leve y +++ crecimiento con cavidad fuerte. Los aislamientos 6-M2, Ech2A, CfspA, Cfsab y Pt1-A provocaron cavidad profunda en el medio CVP (+++). En el caso de las cepas 
Ech2A, Pt1-A y 6-M2 la profundidad de la misma fue leve, estas cepas bacterianas fueron sometidas posteriormente a la verificación de los Postulados de Koch. Por otro lado, los aislamientos Tt_CP_2, Cf1, Cf2, Cf3, Cf4, Cf5, Pt1-B, Pt4-1, Pt4-2 y Cfsa lograron crecer en el medio de CVP pero sin causar la cavidad.

\section{Postulados de Koch para aislamientos seleccionados}

De acuerdo con los síntomas posteriores a la inoculación de las cepas Ech2A, CfspA y Cfsab en frutos de chile se observó que, en el caso de Ech2A, al sexto día de evaluación se presentó un halo de degradación tisular alrededor del punto inoculado con presencia de exudados, mientras que al día diez incrementó la degradación y se formaron laceraciones en la superficie del fruto (Figura 3A y 3B).
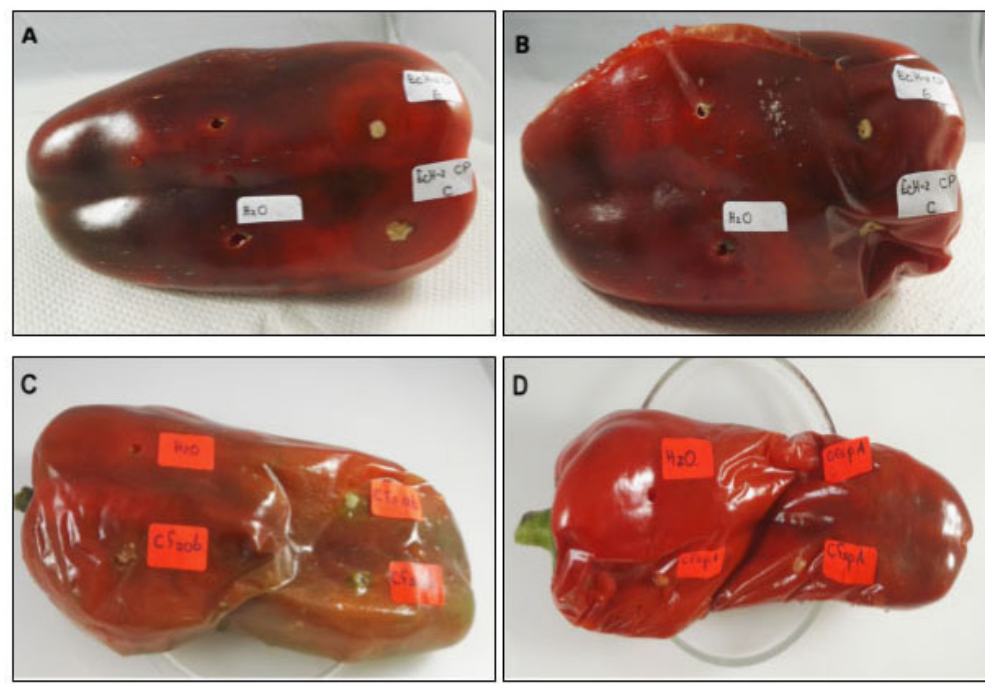

Figura 3. Inoculación de frutos de chile (Capsicum annuum) con tres cepas pectinolíticas obtenidas de frutos de chile con síntomas de pudrición blanda en Cartago y Alajuela. Se inocularon tres puntos con el aislamiento correspondiente y uno con agua estéril como control negativo. A, B. Seis y diez días después de la inoculación con la cepa Ech2A, respectivamente, C. Cuatro días post Inoculación con la cepa CfspA. D. Cuatro días post inoculación con la cepa Cfsab. Laboratorio de Microbiología Ambiental (CIBCM), Universidad de Costa Rica, San José, Costa Rica. 2019.

Figura 3. Inoculation of Capsicum annuum fruits with pectinolytic strains obtained from pepper fruit with soft rot symptoms in Cartago and Alajuela, Costa Rica. Three points were inoculated with the corresponding isolated and one with sterile water as a negative control. A, B. Six and ten days later of inoculation with strain Ech2A, respectively. C. Four days post Inoculation with the CfspA strain. D. Four days post Inoculation with the Cfsab strain. Environmental Microbiology Laboratory (CIBCM), University of Costa Rica, San José, Costa Rica. 2019.

Un día posterior a la inoculación de la cepa CfspA se observó un área decolorada y suave alrededor de los puntos y cuatro días después, degradación del tejido circundante (Figura 3D). El aislamiento Cfsab produjo degradación completa en los puntos al cuarto día de evaluación (Figura 3C). En todos los casos el punto inoculado con agua como control negativo no presentó sintomatología. En el caso de las inoculaciones con las cepas PtA y 6-M2 aisladas de papa y cebolla, respectivamente, no se reprodujo el síntoma en las plantas inoculadas. 


\section{Reaislamiento del patógeno}

De acuerdo con el análisis de tipificación de las cepas causantes de la sintomatología, el patrón de bandas de cada una de las cepas del patógeno bacteriano reaislado a partir de la planta inoculada, presentó un $100 \%$ de similitud con el patrón observado de las cepas CfspA, Cfsab y Ech2A (Figura 4). Este análisis, además de la comparación de las características morfológicas, comprobó que la cepa inoculada y la recuperada fueron la misma, por lo que los postulados de Koch se cumplieron para los aislamientos Ech2A, CfspA y Cfsab en frutos de chile.

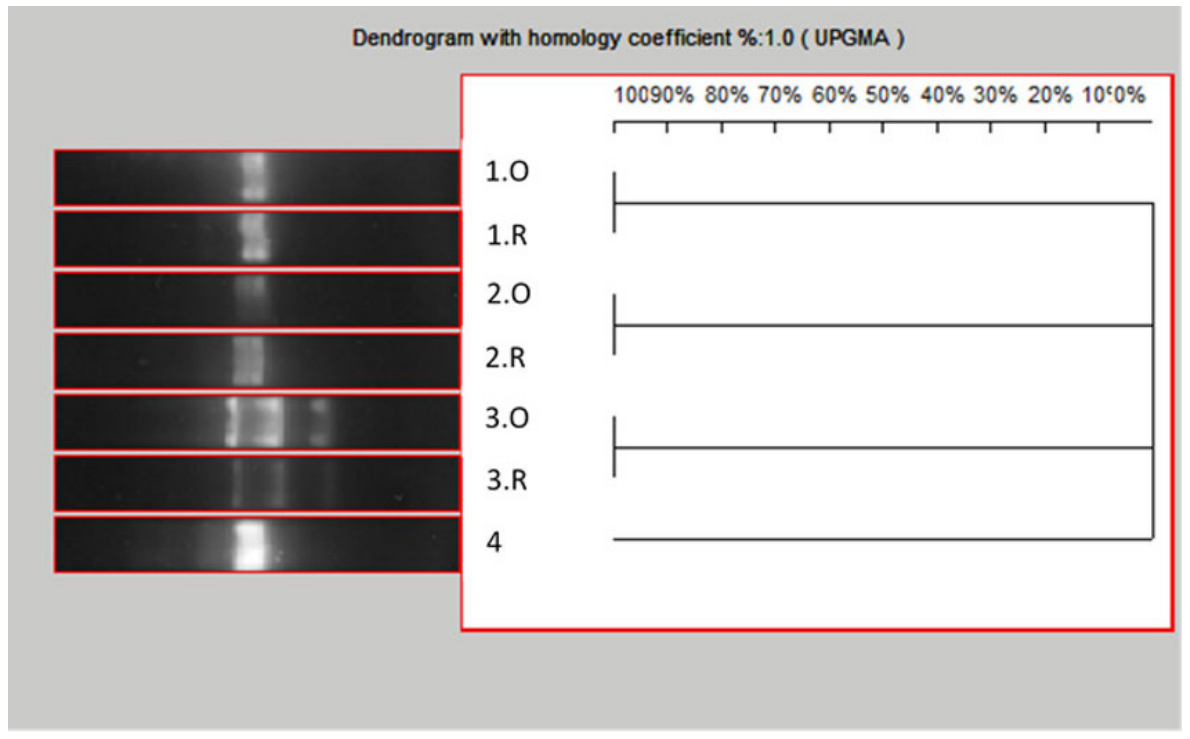

Figura 4. Comparación mediante análisis con base en el método de agrupamiento de pares no ponderados con media aritmética (UPGMA) de la región intergénica 16S-23S del gen rARN (ITS) de bacterias originales y reaisladas para comprobar los postulados de Koch con las cepas 1.O) CfspA inoculada, 1.R) CfspA reaislada, 2.O) Cfsab inoculada, 2.R) Cfsab reaislada, 3.O) Ech2A, 3.I) Ech2A reaislada 4) control positivo (P. carotovorum ATCC 495). Laboratorio de Microbiología Ambiental (CIBCM), Universidad de Costa Rica, San José, Costa Rica. 2019.

Figure 4. UPGMA analysis by comparing the ITS region of original ADN re-isolated bacteria for the verification of Koch postulates with the strains 1.O) CfspA inoculated, 1.R) CfspA re-isolated, 2.O) Cfsab inoculated, 2.R) Cfsab reisolated, 3.O) Ech2A, 3.I) Ech2A reisolated 4) positive control (P. carotovorum ATCC 495). Environmental Microbiology Laboratory (CIBCM), University of Costa Rica, San José, Costa Rica. 2019.

\section{Análisis filogenético}

El árbol obtenido mediante el análisis filogenético del gen ARNr 16S de los aislamientos causantes de los síntomas, estuvo conformado por tres clados compuestos por los géneros Pectobacterium, Dickeya y Raoultella (Figura 5). Las cepas CfspA y Cfsab se agruparon con secuencias tipo del género Pectobacterium y mostraron mayor similitud con P. carotovorum subsp. brasiliense cepa 1073 (Nabhan et al., 2012). A pesar de que ambas cepas fueron aisladas a partir de la misma muestra, el análisis filogenético (Figura 5) indicó que sus secuencias difirieron entre sí. Morfológicamente, CfspA formó colonias blancas, pequeñas y translúcidas en agar nutritivo, mientras que Cfsab forma colonias de mayor tamaño, blancas y opacas, lo cual puede indicar que se tratan de subespecies o cepas diferentes. 


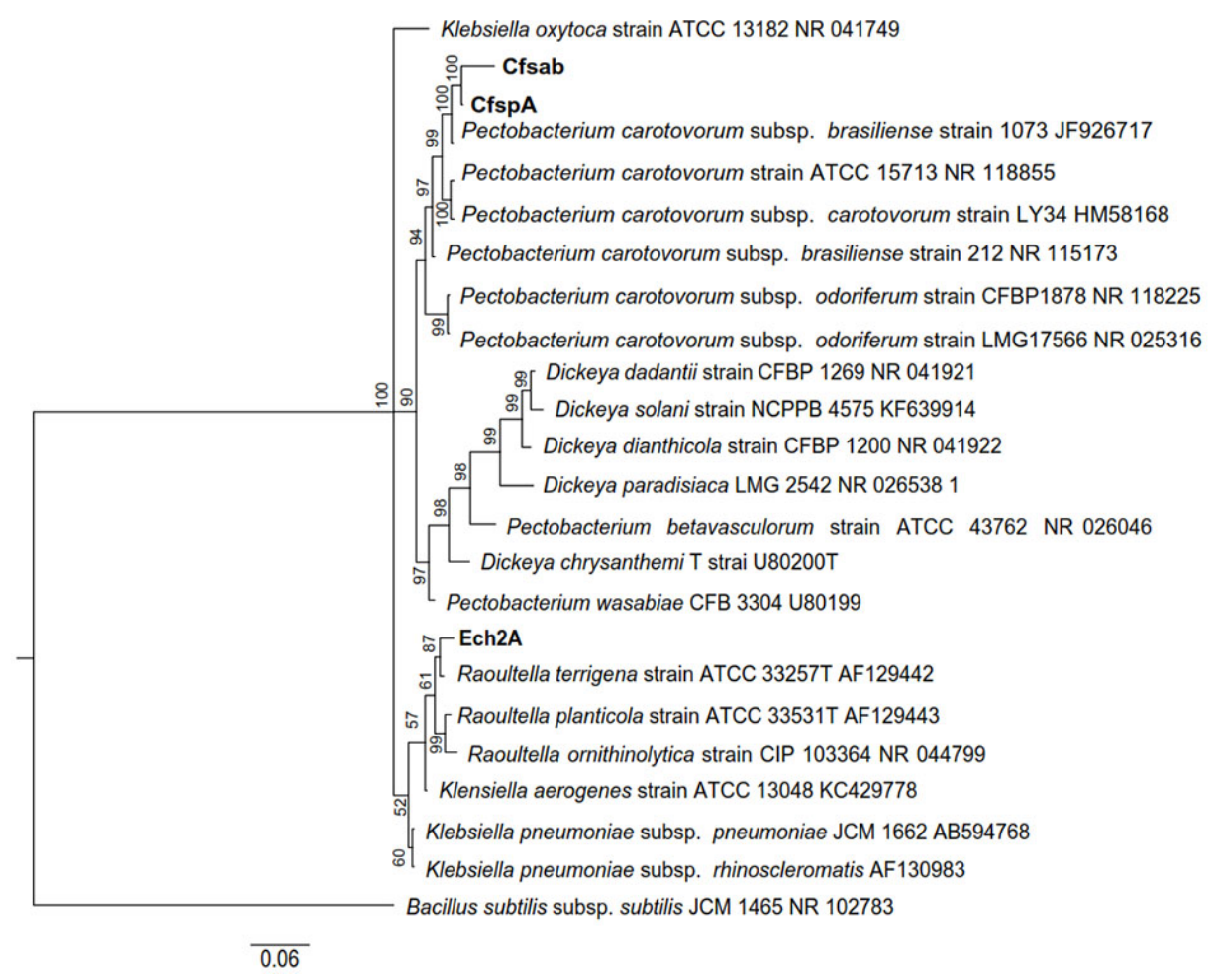

Figura 5. Árbol filogenético bayesiano basado en secuencias del gen ARNr 16S para las cepas pectinolíticas. Se utilizó como grupo externo la secuencia de la cepa tipo Bacillus subtilis subsp. subtilis JMC 1465. Modelo de sustitución TN93+G+I. Laboratorio de Microbiología Ambiental (CIBCM), Universidad de Costa Rica, San José, Costa Rica. 2019.

Figure 5. Bayesian phylogenetic tree based on $16 \mathrm{~S}$ ribosomal sequences for pectinolytic strains. The Bacillus subtilis subsp subtilis JMC 1465 type strain sequence was considered outgroup. Replacement model TN93+G+I. Environmental Microbiology Laboratory (CIBCM), University of Costa Rica, San José, Costa Rica. 2019.

La cepa Ech2A se ubicó dentro del clado de Klebsiella y Raoultella, mostrando mayor similitud (87 \% de remuestreo) con la cepa tipo Raoultella terrígena ATCC 3325T (AF129442) aislada de una muestra de agua potable (Izard et al.,1981).

Las secuencias del gen 16S ARNr para las cepas Ech2A, CfspA y Cfsab se depositaron en la base de datos del GenBank (número de accesión MN386194, MN386195 y MN386196, respectivamente).

\section{Discusión}

La reacción hipersensible positiva es un indicador de patogenicidad de las bacterias fitopatógenos y se utilizó para separarlas de cepas oportunistas. La inducción de la RH por los aislamientos en este estudio sugiere que existió una relación de incompatibilidad planta-patógeno, donde la RH desencadenó la muerte celular programada como una primera línea de defensa ante la detección de organismos patógenos (Klement \& Goodman, 1967; Kushalappa et al., 2016).

Se observaron diferentes grados de intensidad en la RH realizada en las plantas de chile dulce dependiendo de la cepa evaluada, que fueron desde una clorosis baja o débil hasta la necrosis del tejido. Se ha reportado que, de forma similar, algunas cepas de Pectobacterium causaron una RH leve cuando fueron inoculadas en hojas 
de tabaco, esto por la presencia de un efector único y divergente (Pitman et al., 2010). La cantidad de proteínas efectoras sintetizadas por las bacterias juegan un papel en la variabilidad fenotípica de la RH (Barny, 1995; Huang et al., 2004), por lo que se podría sugerir que las cepas que causaron una RH leve produjeron una menor cantidad de proteínas efectoras que las que causaron una reacción alta. La producción baja de estas le permite a la planta contener el avance del patógeno de forma más simple, sin la necesidad de liberar gran cantidad de proteínas de resistencia y sin tener que desencadenar una muerte celular tan drástica en el punto de reconocimiento.

La necrosis provocada por las cepas CfspA y Cfsab en el punto de inoculación se debió probablemente a que fueron aisladas a partir de lesiones en frutos de chile dulce, que corresponden a patógenos de este cultivo y al ser inoculados en las hojas del mismo, pudieron liberar sustancias que interactuaron e inhibieron defensas de la planta, lo que permitió su avance por los tejidos (Etalo et al., 2013). Estas sustancias liberadas por los patógenos bacterianos son conocidas como efectores y dentro de sus acciones para modular las respuestas de las plantas están la degradación y supresión de fitohormonas como el ácido jasmónico y ácido salicílico, supresión de la actividad del proteosoma, modulación de canales de calcio e inactividad de quinasas (Feng \& Zhou, 2012; Asai \& Shirasu, 2015). Además, se ha visto que patógenos liberan efectores que inducen la muerte celular en las plantas, y en el caso de organismos necrotróficos, como las SRB, esta muerte del tejido puede contribuir al avance del patógeno (Govrin \& Levine, 2000; Staats et al., 2005; Kim et al., 2011; Williams et al., 2011; Charkowski et al., 2012; Gupta et al., 2015).

La capacidad de degradar la pectina de los diferentes aislamientos fue comprobada mediante la utilización de placas Petri con medio de cristal violeta y pectato. De los aislamientos sometidos a esta prueba, solo se observó degradación de pectina en los aislamientos Ech2A, 6M2, CfspA, Cfsab y Pt1-A, los cuales lograron causar cavidad en el medio CVP y se observaron cavidades más profundas en las cepas CfspA y Cfsab. Además, se observó el crecimiento superficial de otras cepas, pero sin la formación de cavidades. Es posible que especies de Pseudomonas y Flavobacterium puedan crecer también y formar cavidades, ya que producen enzimas pectinolíticas (Charkowski, 2007). Por otro lado, es posible que ciertas bacterias saprofitas puedan crecer como contaminantes en el medio CVP, sin embargo, no producen la cavidad (Hélias et al., 2012).

Para las cepas 6M2 y Pt1-A no se logró reproducir los síntomas originales en las plantas inoculadas. En el caso de las plantas de papa y cebolla inoculadas con las bacterias, las condiciones de temperatura y humedad no pudieron ser controladas, a pesar de que las plantas se colocaron dentro de cajas plásticas en el caso de papa y en bolsas plásticas para la cebolla, puede que las condiciones propiciadas no fueran las idóneas para que se desarrollara la enfermedad. La alta humedad relativa y temperatura son factores claves para que tanto la infección como el desarrollo de la enfermedad se lleven a cabo en los cultivos (Charkowski, 2007). En el caso de la papa, el factor más importante es el nivel del agua en el suelo, favoreciéndose el desarrollo de la enfermedad en los tubérculos con periodos prolongados de excesiva humedad en el suelo, lo que propicia condiciones anaeróbicas para las bacterias y, a su vez aumentan su multiplicación e invasión (Perombelon \& Kelman, 1980).

En el caso de las cepas Ech2A, CfspA y Cfsab, provenientes de frutos de chile dulce, sí fue posible reproducir los síntomas en los frutos inoculados. Para los tres casos, se observó que en los puntos inoculados con las bacterias el tejido se tornó blando de forma semejante a los síntomas presentados por los frutos de chile de donde fueron aisladas las bacterias (Figura 2), más tarde se dio la aparición de exudados blanquecinos y laceraciones en la superficie de los frutos producto de descomposición del tejido, lo anterior concuerda con los hallazgos realizadas por Gillis et al. (2014) y Estrada et al. (2000) que, al inocular frutos de chile dulce con bacterias de pudrición blanda, observaron lesiones iguales a las descritas anteriormente.

Se observó que para la cepa Ech2A, la aparición de los síntomas fue más lenta que para las cepas CfspA y Cfsab, lo que podría indicar diferentes capacidades de virulencia y agresividad, que podría estar relacionado con la cantidad de enzimas pectinolíticas que producen o con que las condiciones de temperatura y humedad óptimas 
fueron diferentes, debido a que provinieron de dos zonas distintas (Charkowski, 2007; Davidsson et al., 2013; Perombelon \& Kelman, 1980; Toth et al., 2003).

Según el análisis filogenético, la cepa Ech2A se ubicó dentro del mismo clado que secuencias de cepas tipo de Raoultella y Klebsiella (Figura 5), siendo mayor su similitud a la especie Raoultella terrigena. Estos géneros bacterianos se han reportado como aislamientos clínicos, muestras ambientales y organismos benéficos de plantas, en su mayoría, y en el menor de los casos como patógenos de las plantas como zanahoria y banano (Brisse et al., 2006; Brown \& Seidler, 1973; Chandrashekar et al., 2018; Fan et al., 2016; Knittel et al., 1977).

Las secuencias de las cepas CfspA y Cfsab se relacionaron cercanamente con P. carotovorum subsp. brasiliense. Esta especie fue descrita oficialmente en el 2012 por (Nabhan et al., 2012), sin embargo, el nombre brasiliense se utilizó por primera vez en el 2004, cuando Duarte et al. (2004) realizaron un estudio caracterizando cepas atípicas causantes de podredumbre blanda de la papa en Brasil. En su estudio se encontró que la bacteria que causaba esta enfermedad en Brasil difería de las cepas Erwinia carotovora subsp. atroseptica (actualmente Pectobacterium atrosepticum), Erwinia carotovora (actualmente Pectobacterium carotovorum subsp. carotovorum) y Erwinia chrysanthemi (actualmente Dikeya chrysanthemi), las cuales fueron reportadas como las causantes del daño en papa alrededor del mundo. Desde su aislamiento por primera vez en el cultivo de papa, P. carotovorum subsp. brasiliense se ha reportado causando podredumbre blanda en diferentes cultivos como pepino (Meng et al., 2016), remolacha azucarera, col, calabacín (Waleron et al., 2015), tomate (Jaramillo et al., 2016), ornamentales (Ma et al., 2007) y en chile dulce (Choi \& Kim, 2013; Gillis et al., 2017).

\section{Conclusiones}

Las cepas CfspA, Cfsab y Ech2A, aisladas de lesiones de pudrición blanda en chile dulce lograron reproducir los síntomas en este hospedero en ensayos de patogenicidad.

Según los análisis filogenéticos realizados mediante el gen ARNr 16S, las bacterias se clasificaron como $P$. carotovorum subsp. brasiliense y Raoultella terrigena.

A pesar que el género Pectobacterium es un fitopatógeno distribuido mundialmente, esta subespecie no ha sido reportado anteriormente en Costa Rica mediante estudios de patogenicidad y moleculares. En el caso de Raoultella, no existen reportes de esta enterobacteria y su relación con la pudrición blanda, por lo que es necesario realizar más estudios de rango de hospederos, con el fin de determinar si tiene la capacidad de infectar otros cultivos.

\section{Agradecimientos}

Los autores expresan su agradecimiento a la Vicerrectoría de Investigación de la Universidad de Costa Rica quien financió la investigación por medio del proyecto B7200- "Evaluación de la diversidad de la bacteria Pectobacterium carotovorum, agente causal de pudrición blanda en hortalizas y su suceptibilidad a rizobacterias promotoras de crecimiento".

\section{Referencias}

Agrios, G. N. (2005). Plant Pathology (5th ed.). Elsevier.

Arauz, L. (2011). Fitopatología: Un enfoque agroecológico (5 $\left.5^{\mathrm{a}} \mathrm{ed}.\right)$. Editorial Universidad de Costa Rica. 
Asai, S., \& Shirasu, K. (2015). Plant cells under siege: Plant immune system versus pathogen effectors. Current Opinion in Plant Biology, 28, 1-8. https://doi.org/10.1016/j.pbi.2015.08.008

Barny, M. A. (1995). Erwinia amylovora hrpN mutants, blocked in harpin synthesis, express a reduced virulence on host plants and elicit variable hypersensitive reactions on tobacco. European Journal of Plant Pathology, 101(3), 333-340. https://doi.org/10.1007/BF01874789

Bdliya, B. S., \& Langerfeld, E. (2005). A semi-selective medium for detection, isolation and enumeration of Erwinia carotovora ssp. Carotovora from plant materials and soil. Tropical Science, 45(2), 90-96. https://doi.org/10.1002/ts.56

Bhat, K., Masood, S., Bhat, N., Bhat, M. A., Razvi, S., Mir, M., Akhtar, S., Wani, N., \& Habib, M. (2010). Current status of post harvest soft rot in vegetables: A review. Asian Journal of Plant Sciences, 9(4), 200-208. https://doi.org/10.3923/ ajps.2010.200.208

Bonas, U., Schulte, R., Fenselau, S., Minsavage, G. V., Staskawicz, B. J., \& Stall, R. E. (1991). Isolation of a gene cluster from Xanthomonas campestris pv. Vesicatoria that determines pathogenicity and the hypersensitive response on pepper and tomato. Molecular Plant-Microbe Interactions, 4(1), 81-88. https://doi.org/10.1094/mpmi-4-081

Brisse, S., Grimont, F., \& Grimont, P. A. D. (2006). The genus Klebsiella. In M. Dworkin, S. Falkow, E. Rosenberg, K.-H. Schleifer, \& E. Stackebrandt (Eds.), The Prokaryotes: Volume 6: Proteobacteria: Gamma Subclass (pp. 159-196). Springer New York. https://doi.org/10.1007/0-387-30746-X_8

Brown, C., \& Seidler, R. J. (1973). Potential pathogens in the environment: Klebsiella pneumoniae, a taxonomic and ecological enigma. Applied Microbiology, 25(6), 900-904. https://doi.org/10.1128/AEM.25.6.900-904.1973

Byrd, A. L., \& Segre, J. A. (2016). Adapting Koch's postulates. Science, 351(6270), 224-226. https://doi.org/10.1126/science. aad6753

Chandrashekar, B. S., Prasannakumar, M. K., Puneeth, M. E., Teli, K., Priyanka, K., Mahesh, H. B., \& Desai, R. U. (2018). First report of bacterial soft rot of carrot caused by Klebsiella variicola in India. New Disease Reports, 37, Article 21. https://doi.org/10.5197/j.2044-0588.2018.037.021

Charkowski, A.O. (2007). The soft rot Erwinia. In S. S. Gnanamanickam (Ed.), Plant-Associated Bacteria (pp. 423-505). Springer, Dordrecht. https://doi.org/10.1007/978-1-4020-4538-7_13

Charkowski, A. O. (2018). The Changing Face of Bacterial Soft-Rot Diseases. Annual Review of Phytopathology, 56(1), 269-288. https://doi.org/10.1146/annurev-phyto-080417-045906

Charkowski, A., Blanco, C., Condemine, G., Expert, D., Franza, T., Hayes, C., Hugouvieux-Cotte-Pattat, N., Solanilla, E. L., Low, D., Moleleki, L., Pirhonen, M., Pitman, A., Perna, N., Reverchon, S., Rodríguez Palenzuela, P., San Francisco, M., Toth, I., Tsuyumu, S., van der Waals, J., Yedidia, I. (2012). The role of secretion systems and small molecules in soft-rot enterobacteriaceae pathogenicity. Annual Review of Phytopathology, 50(1), 425-449. https://doi.org/10.1146/ annurev-phyto-081211-173013

Charkowski, A. O., Lind, J., \& Rubio-Salazar, I. (2014). Genomics of plant-associated bacteria: The soft rot enterobacteriaceae. In D. C. Gross, A. Lichens-Park, \& C. Kole, (Eds.), Genomics of plant-associated bacteria (pp. 37-58). Springer. https://doi.org/10.1007/978-3-642-55378-3_2

Chinchilla, C., Gonzales, L., \& Morales, F. (1979). Purdrición bacteriana del cogollo de la piña en Costa Rica. Agronomía Costarricense, 3(2), 183-185. 
Choi, O., \& Kim, J. (2013). Pectobacterium carotovorum subsp. Brasiliense Causing Soft Rot on Paprika in Korea. Journal of Phytopathology, 161(2), 125-127. https://doi.org/10.1111/jph.12022

Czajkowski, R., Pérombelon, M. C. M., Jafra, S., Lojkowska, E., Potrykus, M., van der Wolf, J. M., \& Sledz, W. (2015). Detection, identification and differentiation of Pectobacterium and Dickeya species causing potato blackleg and tuber soft rot: A review. Annals of Applied Biology, 166(1), 18-38. https://doi.org/10.1111/aab.12166

Davidsson, P. R., Kariola, T., Niemi, O., \& Palva, T. (2013). Pathogenicity of and plant immunity to soft rot pectobacteria. Frontiers in Plant Science, 4, Article 191. https://doi.org/10.3389/fpls.2013.00191

Duarte, V., Boer, S. H. D., Ward, L. J., \& de Oliveira, A. M. R. (2004). Characterization of atypical Erwinia carotovora strains causing blackleg of potato in Brazil. Journal of Applied Microbiology, 96(3), 535-545. https://doi.org/10.1111/j.13652672.2004.02173.x

Estrada, R. S. G., Reyes, C. J., Fasio, J. A. C., Molar, R. A., Zequera, I. M., \& Rangel, M. D. M. (2000). Marchitez bacteriana en chile bell causada por Erwinia carotovora subsp carotovora. Revista Mexicana de Fitopatología, 18(2), $120-124$.

Etalo, D. W., Stulemeijer, I. J. E., Esse, H. P. van, de Vos, R. C. H., Bouwmeester, H. J., \& Joosten, M. H. A. J. (2013). System-Wide Hypersensitive Response-Associated Transcriptome and Metabolome Reprogramming in Tomato. Plant Physiology, 162(3), 1599-1617. https://doi.org/10.1104/pp.113.217471

Fan, H. C., Zeng, L., Yang, P. W., Guo, Z. X., \& Bai, T. T. (2016). First report of banana soft rot caused by Klebsiella variicola in China. Plant Disease, 100(2), 517-517. https://doi.org/10.1094/PDIS-05-15-0586-PDN

Feng, F., \& Zhou, J. M. (2012). Plant-bacterial pathogen interactions mediated by type III effectors. Current Opinion in Plant Biology, 15(4), 469-476. https://doi.org/10.1016/j.pbi.2012.03.004

Fontecha, G. (2003). Análisis comparativo de las comunidades de procariotas intestinales de Rothschildia lebeau (Lepidoptera) mediante los polimorfismos en los fragmentos terminales de restricción (T-RFLP) del gen ARNr 16S [Tesis de Maestría no publicada]. Universidad de Costa Rica.

Gardan, L., Gouy, C., Christen, R., \& Samson, R. (2003). Elevation of three subspecies of Pectobacterium carotovorum to species level: Pectobacterium atrosepticum sp. nov., Pectobacterium betavasculorum sp. nov. and Pectobacterium wasabiae sp. nov. International Journal of Systematic and Evolutionary Microbiology, 53(2), 381-391. https://doi. org/10.1099/ijs.0.02423-0

Gillis, A., Santana, M. A., Rodríguez, M., \& Romay, G. (2017). First Report of Bell Pepper Soft-Rot Caused by Pectobacterium carotovorum subsp. Brasiliense in Venezuela. Plant Disease, 101(9), 1671-1671. https://doi.org/10.1094/PDIS-0317-0361-PDN

Govrin,E. M., \& Levine, A. (2000). The hypersensitive response facilitates plant infection by the necrotrophic pathogen Botrytis cinerea. Current Biology, 10(13), 751-757. https://doi.org/10.1016/S0960-9822(00)00560-1

Gupta, R., Lee, S. E., Agrawal, G. K., Rakwal, R., Park, S., Wang, Y., \& Kim, S. T. (2015). Understanding the plant-pathogen interactions in the context of proteomics-generated apoplastic proteins inventory. Frontiers in Plant Science, 6, Article 352. https://doi.org/10.3389/fpls.2015.00352

Hall, T. A. (1999). BioEdit: A user-friendly biological sequence alignment editor and analysis program for Windows 95/98/NT. Nucleic Acids Symposium Series, 41, 95-98. https://doi.org/10.14601/Phytopathol_Mediterr-14998u1.29 
Hélias, V., Hamon, P., Huchet, E., Wolf, J. V. D., \& Andrivon, D. (2012). Two new effective semiselective crystal violet pectate media for isolation of Pectobacterium and Dickeya. Plant Pathology, 61(2), 339-345. https://doi.org/10.1111/j.13653059.2011.02508.x

Herrera, J., \& González, L. (1977). Development and control of potato blackleg, caused by Erwinia carotovora var. Atroseptica, in Costa Rica. Agronomía Costarricense, 1(2), 161-163.

Huang, H. E., Ger, M. J., Yip, M. K., Chen, C. Y., Pandey, A. K., \& Feng, T. Y. (2004). A hypersensitive response was induced by virulent bacteria in transgenic tobacco plants overexpressing a plant ferredoxin-like protein (PFLP). Physiological and Molecular Plant Pathology, 64(2), 103-110. https://doi.org/10.1016/j.pmpp.2004.05.005

Hugouvieux-Cotte-Pattat, N., Condemine, G., \& Shevchik, V. E. (2014). Bacterial pectate lyases, structural and functional diversity. Environmental Microbiology Reports, 6(5), 427-440. https://doi.org/10.1111/1758-2229.12166

Izard, D., Ferragut, C., Gavini, F., Kersters, K., De Ley, J., \& Leclerc, H. (1981). Klebsiella terrigena, a New Species from Soil and Water. International Journal of Systematic Bacteriology, 31(2), 116-127. https://doi.org/10.1099/00207713-312-116

Jaramillo, A., Huertas, C. A., \& Gómez, E. D. (2016). First Report of Bacterial Stem Rot of Tomatoes Caused by Pectobacterium carotovorum subsp. Brasiliense in Colombia. Plant Disease, 101(5), 830. https://doi.org/10.1094/PDIS-08-16-1184PDN

Jensen, M. A., Webster, J. A., \& Straus, N. (1993). Rapid identification of bacteria on the basis of polymerase chain reactionamplified ribosomal DNA spacer polymorphisms. Applied and Environmental Microbiology, 59(4), 945-952. https:// doi.org/10.1128/AEM.59.4.945-952.1993

Kim, J. H., Joen, Y. H., Kim, S. G., \& Kim, Y. H. (2007). First report on bacterial soft rot of graft-cactus Chamaecereus silvestrii caused by Pectobacterium carotovorum subsp. Carotovorum in Korea. The Plant Pathology Journal, 23(4), $314-317$. https://doi.org/10.5423/PPJ.2007.23.4.314

Kim, H. S., Thammarat, P., Lommel, S. A., Hogan, C. S., \& Charkowski, A. O. (2011). Pectobacterium carotovorum Elicits Plant Cell Death with DspE/F but the P. carotovorum DspE Does Not Suppress Callose or Induce Expression of Plant Genes Early in Plant-Microbe Interactions. Molecular Plant-Microbe Interactions, 24(7), 773-786. https://doi. org/10.1094/MPMI-06-10-0143

Klement, Z. (1963). Rapid detection of the pathogenicity of phytopathogenic pseudomonads. Nature, 199, 299-300. https://doi. $\operatorname{org} / 10.1038 / 199299 b 0$

Klement, Z., \& Goodman, R. (1967). The hypersensitive reaction to infection by bacterial plant pathogens. Annual Review of Phytopathology, 5(1), 17-44. https://doi.org/10.1146/annurev.py.05.090167.000313

Knittel, M. D., Seidler, R. J., Eby, C., \& Cabe, L. M. (1977). Colonization of the botanical environment by Klebsiella isolates of pathogenic origin. Applied and Environmental Microbiology, 34(5), 557-563. https://doi.org/10.1128/AEM.34.5.557563.1977

Kumar, S., Stecher, G., \& Tamura, K. (2016). MEGA7: Molecular evolutionary genetics analysis version 7.0 for bigger datasets. Molecular Biology and Evolution, 33(7), 1870-1874. https://doi.org/10.1093/molbev/msw054

Kushalappa, A. C., Yogendra, K. N., \& Karre, S. (2016). Plant innate immune response: qualitative and quantitative resistance. Critical Reviews in Plant Sciences, 35(1), 38-55. https://doi.org/10.1080/07352689.2016.1148980 
Lane, D. (1991). 16S/23S rRNA sequencing. In E. Stackebrandt, \& M. Goodfellow (Eds.), Nucleic acid techniques in bacterial systematics (pp. 115-175). John Wiley \& Sons, Inc.

Ma, B., Hibbing, M. E., Kim, H. S., Reedy, R. M., Yedidia, I., Breuer, J., Breuer, J., Glasner, J. D., Perna, N. T., \& Kelman, A. (2007). Host range and molecular phylogenies of the soft rot enterobacterial genera Pectobacterium and Dickeya. Phytopathology, 97(9), 1150-1163. https://doi.org/10.1094/PHYTO-97-9-1150

McFarland, J. (1907). The nephelometer: An instrument for estimating the number of bacteria in suspensions used for calculating the opsonic index and for vaccines. Journal of the American Medical Association, 49(14), 1176-1178. https://doi.org/10.1001/jama.1907.25320140022001f

Meng, X., Chai, A., Shi, Y., Xie, X., Ma, Z., \& Li, B. (2016). Emergence of bacterial soft rot in cucumber caused by Pectobacterium carotovorum subsp. Brasiliense in China. Plant Disease, 101(2), 279-287. https://doi.org/10.1094/ PDIS-05-16-0763-RE

Nabhan, S., Boer, S. H. D., Maiss, E., \& Wydra, K. (2012). Taxonomic relatedness between Pectobacterium carotovorum subsp. Carotovorum, Pectobacterium carotovorum subsp. Odoriferum and Pectobacterium carotovorum subsp. Brasiliense subsp. Nov. Journal of Applied Microbiology, 113(4), 904-913. https://doi.org/10.1111/j.1365-2672.2012.05383.x

Ngadze, E., Coutinho, T. A., \& van der Waals, J. E. (2010). First Report of Soft Rot of Potatoes Caused by Dickeya dadantii in Zimbabwe. Plant Disease, 94(10), 1263-1263. https://doi.org/10.1094/PDIS-05-10-0361

Nguyen-the, C., \& Carlin, F. (1994). The microbiology of minimally processed fresh fruits and vegetables. Critical Reviews in Food Science \& Nutrition, 34(4), 371-401. https://doi.org/10.1080/10408399409527668

Perombelon, M. C., \& Kelman, A. (1980). Ecology of the soft-rot Erwinias. Annual Review of Phytopathology, 18(1), $361-387$. https://doi.org/10.1146/annurev.py.18.090180.002045

Pitman, A. R., Harrow, S. A., \& Visnovsky, S. B. (2010). Genetic characterisation of Pectobacterium wasabiae causing soft rot disease of potato in New Zealand. European Journal of Plant Pathology, 126(3), 423-435. https://doi.org/10.1007/ s10658-009-9551-y

Pitman, A. R., Wright, P. J., Galbraith, M. D., \& Harrow, S. A. (2008). Biochemical and genetic diversity of pectolytic enterobacteria causing soft rot disease of potatoes in New Zealand. Australasian Plant Pathology, 37(6), 559-568. https://doi.org/10.1071/AP08056

Pu, X. M., Zhou, J. N., Lin, B. R., \& Shen, H. F. (2012). First report of bacterial foot rot of rice caused by a Dickeya zeae in China. Plant Disease, 96(12), 1818-1818. https://doi.org/10.1094/PDIS-03-12-0315-PDN

Ritchie, D. (2000). Bacterial spot of pepper and tomato. The Plant Health Instructor. The American Phytopathological Society. https://doi.org/10.1094. PHI-I-2000-1027-01.

Ronquist, F., Teslenko, M., van der Mark, P., Ayres, D. L., Darling, A., Höhna, S., Larget, B., Liu, L., Suchard, M. A., \& Huelsenbeck, J. P. (2012). MrBayes 3.2: Efficient Bayesian Phylogenetic Inference and Model Choice Across a Large Model Space. Systematic Biology, 61(3), 539-542. https://doi.org/10.1093/sysbio/sys029

Sánchez, K. R., Zúñiga, O. C., Meneses, M. B., \& González, A. Q. (2019). Etiología de las pudriciones en el tallo de Hylocereus costaricensis, provocadas por Enterobacter hormaechei, en Costa Rica. Agronomía Costarricense, 43(2), 61-73. https://doi.org/10.15517/rac.v43i2.37949 
Schaad, N. W., Jones, J. B., \& Chun, W. (2001). Laboratory guide for the identification of plant pathogenic bacteria. American Phytopathological Society Press.

Singh, J., \& Kaur, L. (2016). Advances in potato chemistry and technology ( $2^{\text {nd }}$ ed.). Academic press.

Staats, M., van Baarlen, P., \& van Kan, J. A. L. (2005). Molecular Phylogeny of the Plant Pathogenic Genus Botrytis and the Evolution of Host Specificity. Molecular Biology and Evolution, 22(2), 333-346. https://doi.org/10.1093/molbev/ msi020

Toth, I. K., Bell, K. S., Holeva, M. C., \& Birch, P. R. J. (2003). Soft rot Erwiniae: From genes to genomes. Molecular Plant Pathology, 4(1), 17-30. https://doi.org/10.1046/j.1364-3703.2003.00149.x

Umesha, S., Richardson, P. A., Kong, P., \& Hong, C. X. (2008). A novel indicator plant to test the hypersensitivity of phytopathogenic bacteria. Journal of Microbiological Methods, 72(1), 95-97. https://doi.org/10.1016/j. mimet.2007.11.002

van-der-Merwe, J. J., Coutinho, T. A., Korsten, L., \& van der Waals, J. E. (2010). Pectobacterium carotovorum subsp. Brasiliensis causing blackleg on potatoes in South Africa. European Journal of Plant Pathology, 126(2), $175-185$. https://doi.org/10.1007/s10658-009-9531-2

Volcy, C. (2008). Genesis and evolution of Koch postulates and their relationship with phytopathology. A review. Agronomía Colombiana, 26(1), 107-115.

Waleron, M., Waleron, K., \& Lojkowska, E. (2015). First Report of Pectobacterium carotovorum subsp. Brasiliense causing soft rot on potato and other vegetables in Poland. Plant Disease, 99(9), 1271-1271. https://doi.org/10.1094/PDIS-0215-0180-PDN

Wei-Salas, S., \& Durán-Quirós, A. (2015). Characterization of land use in the main agricultural areas of the Major Metropolitan Area of Costa Rica. Agronomía Costarricense, 39(1), 151-160.

Williams, B., Kabbage, M., Kim, H.-J., Britt, R., \& Dickman, M. B. (2011). Tipping the balance: Sclerotinia sclerotiorum secreted oxalic acid suppresses host defenses by manipulating the host redox environment. PLOS Pathogens, 7(6), e1002107. https://doi.org/10.1371/journal.ppat.1002107

Wright, P., Triggs, C., \& Burge, G. (2005). Control of bacterial soft rot of calla (Zantedeschia spp.) by pathogen exclusion, elimination and removal. New Zealand Journal of Crop and Horticultural Science, 33(2), 117-123. https://doi.org/1 $0.1080 / 01140671.2005 .9514340$

Zhang, J., Shen, H., Pu, X., Lin, B., \& Hu, J. (2014). Identification of Dickeya zeae as a Causal Agent of Bacterial Soft Rot in Banana in China. Plant Disease, 98(4), 436-442. https://doi.org/10.1094/PDIS-07-13-0711-RE 